\title{
Honey dressing on a leg ulcer with tendon exposure in a patient with type 2 diabetes
}

\author{
Ilaria Teobaldi', Vincenzo Stoico', Fabrizia Perrone', Massimiliano Bruti2 ${ }^{2}$ Enzo Bonora' \\ and Alessandro Mantovani ${ }^{1}$
}

1Division of Endocrinology Diabetes and Metabolism, Department of Medicine and 2Division of Plastic Surgery, Department of Surgery, University and Azienda Ospedaliera Universitaria Integrata of Verona, Verona, Italy
Correspondence

should be addressed

to A Mantovani

Email

alessandro.mantovani24@

gmail.com

\section{Summary}

Honey has been used as a wound dressing for hundreds of years by ancient civilizations, but only recently it has acquired scientific interest because of its relevant biological properties. In the last decade, indeed, several trials and observational studies have reported that, compared to conventional treatment (e.g. antiseptics, polyurethane film, paraffin gauze, soframycin-impregnated gauze), honey dressings seem to be better in healing time of different types of wounds, including diabetic foot ulcers. However, to date, information about a potential favorable biological effect of honey dressings on diabetic ulcers with exposed tendon are still scarce. Notably, foot or leg ulcers with exposed tendon are serious complications in patients with type 2 diabetes, as they are associated with an increased risk of adverse outcome. Therefore, the use of effective and safe treatments to bring these lesions to timely healing is very important in clinical practice. We herein report the case of a Caucasian adult patient with type 2 diabetes presenting a chronic right posterior lower limb ulcer (Texas University Classification (TUC) 2D) with tendon exposure that was successfully treated with honey dressings (glucose oxidase (GOX) positive with peroxide activity) in addition to systemic antibiotic therapy, surgical toilette and skin graft. In our case, the use of honey dressing for treating exposed tendon tissue probably allowed the timely wound healing. Although further studies are required, such treatment may constitute part of the comprehensive management of diabetic wounds, including those with tendon exposure, and should be considered by clinicians in clinical practice.

\section{Learning points:}

- Honey has been used as a wound dressing for hundreds of years, but only recently it has acquired scientific interest for its biological properties.

- Several studies have documented that, compared to conventional dressings, honey seems to be better in healing time of different types of wounds, including diabetic foot ulcers.

- Our case report is the first to highlight the importance to use honey dressings also for the treatment of ulcers with tendon exposure in patients with type 2 diabetes, suggesting that this kind of dressing should be considered by clinicians in clinical practice.

\section{Introduction}

Honey is a viscous, supersaturated sugar solution derived from nectar assembled and worked by honeybees (i.e., Apis mellifera), which contains approximately $40 \%$ of fructose, $30 \%$ of glucose, $20 \%$ of water and $5 \%$ of sucrose, as well as many bioactive substances, including amino acids, vitamins, minerals and enzymes $(1,2)$.

Honey has been used as a wound dressing for hundreds of years by numerous ancient civilizations, but only recently it has acquired scientific interest because 
of its biological properties (for a detailed review on this topic please see 2). Indeed, it is established that honey is able to promote healing processes of wounds through multiple mechanisms $(1,2)$. For instance, several experimental studies have documented that the relative acidity of honey may help the deliverance of oxygen from hemoglobin, thus making the wound environment less advantageous to the bacterial growth (2). The high osmolarity of honey may even draw fluid out of the wound bed in order to promote an outflow of lymph as happens during negative pressure wound therapy (2). In addition, honey has an important broad-spectrum antibacterial activity, although there is difference in efficacy among specific honeys (2).

In the last decade, given the aforementioned biological properties of honey, several trials and observational studies have reported that, compared to other conventional treatment (e.g., antiseptics and gauze), honey dressings appear to be better in healing time of different wounds $(2,3,4,5,6,7)$. In addition, although apparently in a counter-intuitive manner, almost identical results were observed for foot ulcers in patients with type 2 diabetes $(3,4)$. However, to date, information regarding the potential favorable biological effects of honey dressings on ulcers with exposed tendon are still scarce, especially in those with type 2 diabetes mellitus. Importantly, foot or leg ulcers with exposed tendon are serious complications in such patients, as they are strongly associated with an increased risk of adverse outcome and even limb amputation (8). In this context, a retrospective observational study of nearly 400 patients with type 2 diabetes underwent surgical treatment for diabetic foot syndrome $(8 \%$ of whom underwent immediate surgical debridement resulting in exposed tendon) shows the importance to cover an exposed and (still) healthy tendon as soon as possible, in order to avoid a rapid spread of infection and also preserving maximal residual foot length for future potential rehabilitation and mobility (9).

Given the multiple bioactivities of honey, although more information is at present required, it is reasonable to suppose that honey dressings may be effectively considered for the treatment of leg ulcers with exposed tendon in individuals with type 2 diabetes.

Thus, we herein report the case of a Caucasian adult patient with type 2 diabetes, presenting a chronic right posterior lower limb ulcer (Texas University Classification (TUC) 2D) with tendon exposure that was treated with honey dressings in addition to systemic antibiotic therapy, surgical toilette and skin graft.

\section{Case presentation}

A 79-year-old Caucasian male patient with type 2 diabetes was admitted to our department for the worsening of a leg ulcer. The diagnosis of type 2 diabetes was made approximately 35 years ago and a treatment with insulin (lispro plus glargine) was initiated in 2003. The glycemic compensation, however, was often poor (i.e., hemoglobin A1c was regularly more than $60 \mathrm{mmol} / \mathrm{mol} \mathrm{Hb}$ ).

In addition to type 2 diabetes, his medical history included chronic kidney disease, hypertension, dyslipidemia, retinopathy, distal symmetric sensitivemotor neuropathy and peripheral arteriopathy. The patient was not a smoker. To note, in 1975, the patient had a car crash that caused a right tibia and fibula fracture complicated by osteomyelitis followed by a subsequent limb deformity and skin chronic dystrophy.

The right posterior lower limb ulcer appeared in July 2017, following a domestic trauma, but the patient arrived in our Foot Clinic in November 2017, presenting a lesion of size of $62 \times 51 \mathrm{~mm}$ with tendon exposure, mild perilesional redness and moderate exudate (Fig. 1, Panel A). Given the characteristics of the leg ulcer, we decided to perform a deep biopsy that revealed the presence of a polimicrobic infection, caused by methicillin-sensitive Staphylococcus aureus, Klebsiella oxytoca and Morganella morganii. Therefore, a systemic antibiotic therapy with piperacillina/ tazobactam was started.

\section{Investigation}

The blood tests revealed white blood cells (WBC): $6.4 \times 10^{9} / \mathrm{L}$ (normal range: 4.3-10), hemoglobin: $136 \mathrm{~g} / \mathrm{L}$ (normal range: 135-170), platelets: $196 \times 100$ 000/ $\mathrm{mm}^{3}$ (normal range: 150-450), fasting plasma glucose: $8.9 \mathrm{mmol} / \mathrm{L}$ (normal range: 3.5-5.5), hemoglobin A1c: $78 \mathrm{mmol} / \mathrm{mol} \mathrm{Hb}$ (normal range: <42), alanine aminotransferase (ALT): 28U/L (normal range: 6-50), gamma-glutamyl transpeptidase (GGT): $33 \mathrm{U} / \mathrm{L}$ (normal range: 4-60), creatinine: $136 \mu \mathrm{mol} / \mathrm{L}$ (normal range: 53-115) and C-reactive protein (CRP): $24 \mathrm{mg} / \mathrm{L}$ (normal range: $<5)$.

In addition, in order to complete the diagnostictherapeutic process, a lower limbs arteriosus ultrasonography confirmed the presence of serious peripheral arteriopathy that required an angioplasty of the right anterior tibial artery, which was performed with success. 
Panel A

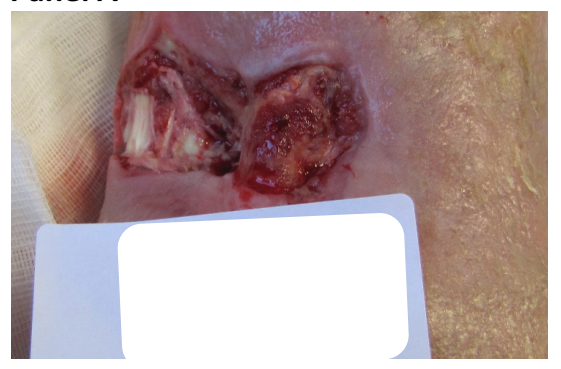

Panel D

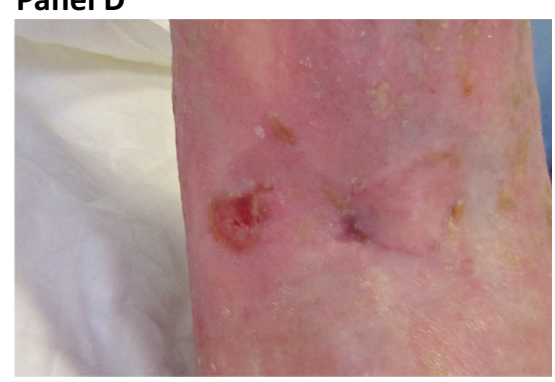

Panel B

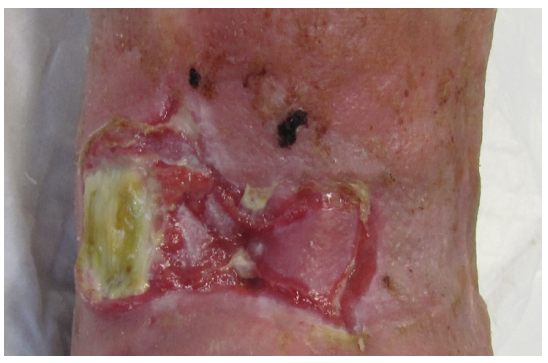

Panel E

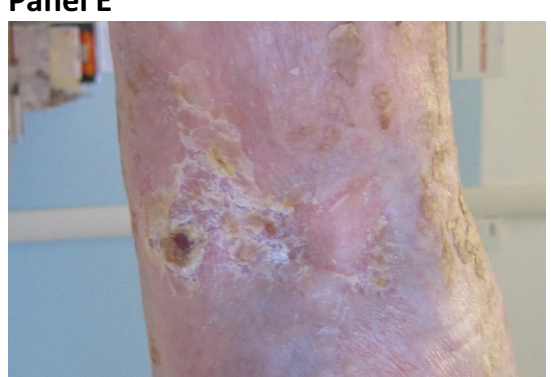

Panel C

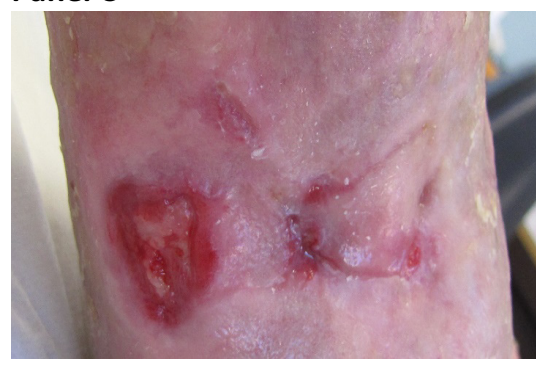

Figure 1

Leg ulcer with tendon exposure in a patient with type 2 diabetes. Panel A, initially the leg ulcer measured $62 \times 51 \mathrm{~mm}$ and was characterized by tendon exposure, mild perilesional redness and moderate exudate. Panel B, after surgical toilette and the skin graft, the tendon exposure persisted, but it was still vital. Panels C, D and E, the use of honey dressing (glucose oxidase positive with peroxide activity) started to promote the re-epithelization of the tendon and the healing of the remaining ulcer. The complete healing of the lesion occurred after 3 months of treatment with the honey dressing.

\section{Treatment}

During hospitalization, the clinical case was extensively discussed with the plastic surgeon and the infectivologist. The first suggested the execution of a surgical toilet followed by a skin graft in order to accelerate the healing, while the second indicated to continue the systemic antibiotic therapy with piperacillina/tazobactam for a total of 5-6 weeks. After the discharge, the skin graft adhesion was less than 50\% and the tendon exposure persisted, but it was still vital (Fig. 1, Panel B).

\section{Outcome and follow-up}

In our ambulatory setting, we continued weekly soft mechanical debridement and conventional antiseptic dressings for at least 5 weeks without particular benefit; in other words, the ulcer was substantially the same size and the tendon was continuously exposed. Subsequently, given the impossibility of healing the lesion with this treatment, we decided to use a dressing based on honey and collagen (i.e., 60\% collagen and $40 \%$ honey) on the top of the vital tendon and a 100\% honey gel on the remaining lower limb ulcer with a weekly frequency. In this case, we used a positive glucose oxidase (GOX) with hydrogen peroxide activity (3\%o). As covering dressing, we used sterile gauzes that were enough to handle the modest exudate produced weekly and following a weakly compressive bandage. The purpose of this treatment was to promote the migration of fibroblasts and other cells as well as to create a relatively acid $\mathrm{pH}$ environment that hampers the bacterial proliferation. A detailed elucidation of the honey dressing was given to the patient. His consent was obtained verbally. By contrast, the patient refused the proposal to use devices or pinstripe splints in order to reduce the supine pressure and the tendon sliding. During a period of approximately 8 weeks, more than half of the ulcer was completely epithelized and after another 10 weeks, the tendon was completely covered (Fig. 1, Panel C). The patient continued dressings with honey balm (composed by $50 \%$ of honey and $50 \%$ of beeswax) twice a week for further 4 weeks, in order to moisturize and soften the wound and the skin around (Fig. 1, Panels D and E).

\section{Discussion}

Honey is a low-cost topical dressing with important beneficial properties for the healing of wounds. Although honey was used as a wound dressing in the past, only recently it has re-acquired scientific interest because of its antimicrobial properties, anti-inflammatory and 
anti-oxidant activity and relevant nutritional contents $(1,2)$. In addition, recent experimental studies have clearly showed that the wound-healing properties of honey may also include the stimulation of tissue growth, the induction of re-epithelialization and the minimized scar formation $(1,2)$.

Our patient was a 79-year-old Caucasian male patient affected by type 2 diabetes presenting a leg infected ulcer with tendon exposure. The presence of an ulcer with tendon exposure in a patient with type 2 diabetes should be always considered with caution, as it is strongly associated with adverse outcomes $(8,9)$. Notably, the tendon was still vital. In our case, the systemic antibiotic therapy, the revascularization of lower limb arteries and the surgical toilette with skin graft have strongly contributed to the improvement of ulcer. However, all these treatments were not sufficient and decisive, as, after discharge, an ulcer of reduced size (about half of the initial one) with a part of the exposed tendon persisted again. At the beginning, in our ambulatory setting, we treated this lesion using conventional treatment (e.g., antiseptics) for several weeks, observing a slow and poor improvement (notably, a relative therapeutic inertia may be observed in this case). For this reason, we decided to change the dressing and to use a honey dressing, seeing a progressive re-epithelization of the tendon and the remaining leg ulcer. Given the improvement of the lesion through the honey dressing, it is possible to speculate that the significant re-epithelization of the lesion may have been determined by the specific biological properties of honey. Obviously, since this is merely a clinical case, we cannot draw any kind of conclusion, but only hypothesize that the antimicrobial, anti-inflammatory, anti-oxidant properties of honey, as well as its bioactive substances (of note, the honey dressing that we used was composed by a high concentration of enzymes, such as bee-defensin 1 (which has an antibacterial action) and glucose oxidase (which promotes the transformation of glucose into hydrogen peroxide, thus concurring to create an acidic milieu)), may have helped to create a favorable environment for the re-epithelization of tendon and healing of the remaining ulcer. This hypothesis is, however, supported by some recent evidence, suggesting that honey dressings may stimulate the immune response, suppress the local inflammation and bring about rapid autolytic debridement, thus promoting the growth of tissues for wound repair $(1,2,3,4,5,6)$. For instance, in a Cochrane systematic review, Jull et al. substantiated that honey dressings seemed to heal partial thickness burns more quickly than conventional treatment (i.e., polyurethane film, paraffin gauze, soframycinimpregnated gauze, sterile linen and leaving the burns exposed), as well as infected post-operative wounds more swiftly than antiseptics and gauze (3). Additionally, in a randomized, controlled trial enrolling 348 type 2 diabetic patients with Wagner's grade 1 and 2 ulcers randomized to honey dressing $(n=179)$ or normal saline dressing, Imran et al. reported that 136 wounds $(75.9 \%)$ out of 179 were completely healed with honey dressing and 97 (57.4\%) out of 169 with saline dressing $(P<0.01)$, thus suggesting that honey was an effective dressing agent instead of conventional dressings in treating diabetic patients with foot ulcer (4).

In conclusion, our case report is clinically relevant, as it highlights the use of honey dressing (as a possible alternative to currently available, often expensive, dressings) in a leg ulcer with exposure of tendon in a patient with type 2 diabetes. Seen that honey includes multiple bioactive molecules, clinicians may consider this kind of dressing in their daily choices of local therapy, also in the presence of tendon exposure. In this case, in fact, it is really important to maintain the moisture balance, especially at the level of the tendon and, at the same time, to avoid contamination and infection that may extent along the tendon and respective compartment, thus worsening the prognosis.

\section{Declaration of interest}

The authors declare that there is no conflict of interest that could be perceived as prejudicing the impartiality of the research reported.

\section{Funding}

This research did not receive any specific grant from any funding agency in the public, commercial or not-for-profit sector.

\section{Patient consent}

Written informed consent has been obtained from the patient for the publication of this report.

\section{References}

1 Eddy JJ, Gideonsen MD \& Mack GP. Practical considerations of using topical honey for neuropathic diabetic foot ulcers: a review. Wisconsin Medical Journal 2008107 187-190.

2 Molan P \& Rhodes T. Honey: a biologic wound dressing. Wounds 201527 141-151.

3 Jull AB, Cullum N, Dumville JC, Westby MJ, Deshpande S \& Walker N. Honey as a topical treatment for wounds. Cochrane Database of Systematic Reviews 2015 CD005083. (https://doi. org/10.1002/14651858.CD005083.pub4) 
4 Imran M, Hussain MB \& Baig M. A randomized, controlled clinical trial of honey-impregnated dressing for treating diabetic foot ulcer. Journal of College of Physicians and Surgeons Pakistan 201525 721-725. (https://doi.org/10.2015/JCPSP.721725)

5 Moghazy AM, Shams ME, Adly OA, Abbas AH, El-Badawy MA, Elsakka DM, Hassan SA, Abdelmohsen WS, Ali OS \& Mohamed BA. The clinical and cost effectiveness of bee honey dressing in the treatment of diabetic foot ulcers. Diabetes Research and Clinical Practice 201089 276-281. (https://doi.org/10.1016/j. diabres.2010.05.021)

6 Al-Waili NS, Salom K \& Al-Ghamdi AA. Honey for wound healing, ulcers, and burns; data supporting its use in clinical practice. Scientific World Journal 201111 766-787. (https://doi.org/10.1100/ tsw.2011.78)
7 Greer N, Foman NA, MacDonald R, Dorrian J, Fitzgerald P, Rutks I $\&$ Wilt TJ. Advanced wound care therapies for nonhealing diabetic, venous, and arterial ulcers a systematic review. Annals of Internal Medicine 2013159 532-542. (https://doi.org/10.7326/0003-4819159-8-201310150-00006)

8 Armstrong DG, Lavery LA \& Harkless LB. Validation of a diabetic wound classification system. The contribution of depth, infection, and ischemia to risk of amputation. Diabetes Care 199821 855-859. (https://doi.org/10.2337/diacare.21.5.855)

9 Clerici G, Caminiti M, Curci V, Quarantiello A \& Faglia E. The use of a dermal substitute to preserve maximal foot length in diabetic foot wounds with tendon and bone exposure following urgent surgical debridement for acute infection. International Wound Journal 20107 176-183. (https://doi.org/10.1111/j.1742-481X.2010.00670.x)

Received in final form 26 September 2018 Accepted 25 October 2018 